\title{
Programa de normalización de estudios previos y control de calidad en las intervenciones:
}

\section{Propiedades Hídricas. I Parte}

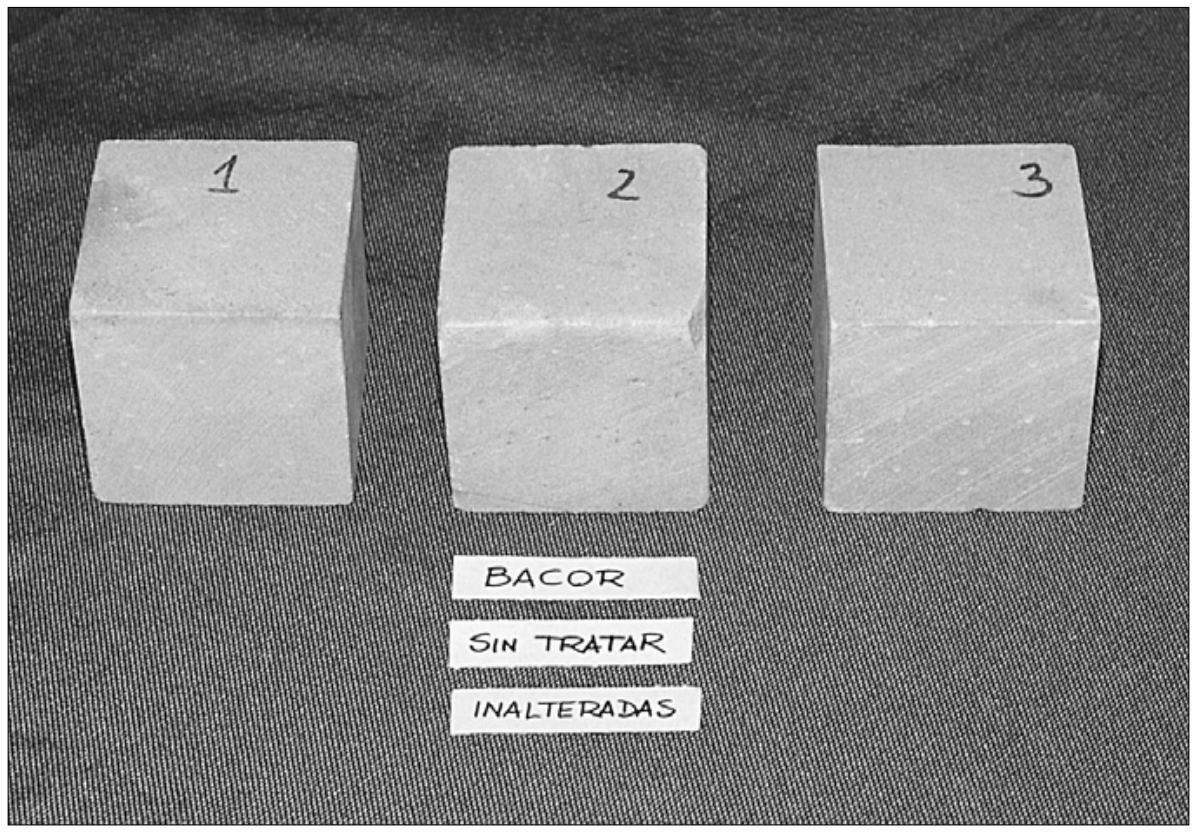

Esther Ontiveros Ortega

Rosario Villegas Sánchez Departamento de análisis del IAPH

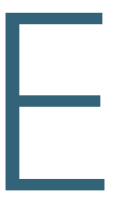

I Subprograma de Normalización de Estudios Previos que se está llevando a cabo en el IAPH pretende realizar una propuesta y descripción de las técnicas de análisis y ensayo que se deben utilizar para el conocimiento de los materiales en general (Boletín IAPH n ${ }^{\circ}$ I5), comenzando por los utilizados en Bienes Inmuebles.

El agua es uno de los agentes de degradación más importante que afectan a la durabilidad de los materiales; es, por tanto, de gran importancia caracterizar y cuantificar el comportamiento hídrico de cualquier material que se haya o pueda ser empleado en la construcción o restauración del Patrimonio Histórico. En este sentido es indispensable conocer la dinámica del movimiento del agua en el seno poroso de estos materiales, porque el agua es, sin duda, una vía importante de transporte de agentes de deterioro, además de un factor de alteración por sí misma. 
El estudio de la porosidad (muy ligado al comportamiento hídrico) resulta de gran interés por varias razones: en ella se produce el almacenamiento y transporte de fluidos y de alguna manera controla las propiedades mecánicas de los materiales. Ésta puede venir dada en forma de poros, -intragranular, intergranular, matricial, intercristalina, móldica y vascular- o fisuras -intragranular, borde de grano y transgranular-.

La humedad existente en los materiales puede proceder de distintas fuentes: humedad atmosférica, agua de precipitación, agua directa procedente de salpicaduras y transporte al interior por capilaridad (penetra por cubiertas, fachadas, juntas y grietas), agua del subsuelo (ascenso capilar del agua en muros de sótanos y plantas bajas), filtraciones de conducciones rotas y humedad de construcción.

El agua en el interior de los materiales se puede encontrar como:

- Agua de constitución. Este agua se caracteriza por reaccionar químicamente con el sólido (entra a formar parte de su estructura cristalina). La presencia de uno u otro estado de hidratación es función de las características del ambiente y de las condiciones de cristalización de cada uno de ellos.

- Agua de adsorción. Se trata de agua ligada a la superficie de los cuerpos cristalinos y, por tanto, relacionada con la superficie específica que poseen los materiales porosos.

- Agua capilar. También llamada de absorción capilar y condensación. Cuando la absorción tiene lugar en un ambiente saturado en vapor de agua ( humedad relativa $>98 \%$ ) y la roca posee poros de tamaño adecuado se producen fenómenos de condensación capilar. Si el sólido se encuentra directamente en contacto con el agua líquida, todos estos poros capilares son rápidamente saturados de agua, hablándose entonces de absorción capilar.

- Agua gravitacional. Este agua puede moverse dentro del medio poroso de acuerdo con las fuerzas que actúan sobre ella (gravedad, presiones externas, etc.), siendo común en la naturaleza el movimiento descendente. En edafología se distingue entre "agua de flujo lento" (la que discurre lentamente por poros de tamaño entre 10 y $50 \mu \mathrm{m}$ ) y "agua de flujo rápido" (la que circula por poros $>50 \mu \mathrm{m}$ ).

En ambientes muy húmedos y en relación con características específicas de los sólidos absorbentes, la absorción de vapor de agua puede dar lugar a nuevos fenómenos:

- Solvatación. Fenómeno característico de algunos sólidos iónicos (ciertas sales) que pueden absorber vapor de agua y pasar de estado anhidro al hidratado, y continuar la absorción hasta total disolución del mismo.

- Hinchamiento. Este fenómeno tiene lugar en materiales de carácter arcilloso, donde la absorción puede proseguir indefinidamente hasta dispersión coloidal.

Las propiedades del agua líquida situada en contacto con materiales sólidos puede ser considerada bajo dos puntos de vista: cantidad de agua retenida por la piedra y movilidad del agua frente a las fases sólidas, ésta movilidad se expresa como la variación del grado de saturación respecto al tiempo.

Para la determinación del comportamiento hídrico de los materiales se emplean una serie de ensayos en los cuales se determinan el contenido en humedad y grado de saturación, que vienen indicados a continuación:

- Absorción forzada de agua.

- Absorción libre de agua por inmersión total.

- Absorción de agua por capilaridad, Succión capilar, Ascenso capilar.

- Absorción de agua a baja presión.

- Absorción de vapor de agua, Higroscopicidad.

- Desorción libre de agua, Evaporación.

- Absorción de gotas: tiempo y ángulo de contacto.

- Permeabilidad al vapor de agua.

Para la descripción de estos ensayos se han seguido las normas siguientes:

UNE: 22-172/85, 22-|82/85, 22-19|/85.

Rilem: (1980) Recomended test to measure the deterioration of stone and assess the efectiveness of treatement methods. Commision 25-PEM. Protection et Erosion des Monument. pp I75-253.

Normal: 44/93, 4l/93, I I/85, 7/81.

\section{PREPARACIÓN DE LAS MUESTRAS}

Aunque los ensayos están fundamentalmente enfocados para material pétreo tanto de carácter natural como artificial (ladrillos, morteros etc,) pueden ser aplicables a cualquier otro tipo de material.

En la caracterización hídrica de materiales empleados en Bienes Inmuebles se utiliza en la mayoría de los casos material procedente de cantera, ya que generalmente es imposible extraer el material necesario de edificios u objetos de interés histórico. En los casos que sea posible es recomendable utilizar material de monumento.

El número de muestras necesarias depende fundamentalmente de la homogeneidad del material, la mayoría de las normativas relacionadas con este tema establecen un número mínimo de dos a tres muestras para el mismo litotipo. En las presentes recomendaciones se fija un número mínimo de tres muestras.

El tamaño de las muestras puede estar condicionado por el tipo de material. Para el caso del material pétreo su dimensión va a estar en función de su textura, en general se recomienda un tamaño diez veces superior a las dimensiones del elemento textural de mayor dimensión. En todos los casos se utilizan muestras de forma geométrica regular normalizadas (cubos, paralelepípedos, cilindros, discos, etc). 


$\begin{array}{ll}\text { Cilindros: } & \text { Diámetro } 5 \mathrm{~cm} \text {, altura } 10 \mathrm{~cm} \\ \text { Discos: } & \text { Diámetro } 5 \mathrm{~cm} \text {, altura } 1 \mathrm{~cm} \\ \text { Prismas: } & 11 \times 5 \times 5 \mathrm{~cm} \\ \text { Cubos: } & 5 \times 5 \times 5 \mathrm{~cm} \\ \text { Lajas: } & 5 \times 5 \times 1 \mathrm{~cm}\end{array}$

Una vez cortadas las muestras deben lavarse con agua destilada para eliminar restos de material pulverulento.

En general es necesario determinar el peso seco de las probetas para lo cual se secarán en estufa a $105^{\circ} \mathrm{C}$, en el caso de muestras sin tratar, y a $60^{\circ} \mathrm{C}$, para muestras tratadas, durante el tiempo necesario (normalmente 48 horas) para obtener peso constante, es decir, que las dos últimas pesadas no varíen más del $0,1 \%$.

\section{ABSORCIÓN FORZADA DE AGUA A VACÍO}

Este ensayo también se denomina absorción de agua al vacío y contenido en agua en Saturación. La finalidad de este ensayo es conocer el contenido máximo de agua que es capaz de retener una roca. A partir de la absorción de agua al vacío se determina la masa saturada $M_{s}$, y puede calcularse el contenido de agua en saturación $W_{S}$ y por tanto el grado de saturación Si.

Además del ensayo aquí propuesto existen numerosos métodos para alcanzar la saturación total de un sólido poroso entre los que destacan:

- Inmersión en agua en ebullición.

- Inmersión en agua a presión elevada.

\section{Procedimiento de ensayo}

Las muestras se secan y se les hace el vacío de 10 a 14 horas; sin romper el vacío se introduce lentamente agua (una hora aproximadamente) hasta cubrirlas totalmente. Se mantiene la absorción de agua bajo vacío otras 10 horas. Ya sin vacío se mantiene la absorción de 24 a 36 horas más, finalmente se determina el peso saturado, Ms, limpiando el exceso de agua en superficie con un paño húmedo. La pesada debe realizarse con una precisión de $0,01 \mathrm{~g}$.

Además del peso seco y saturado, en este ensayo se puede obtener el peso saturado sumergido, $M_{h}$, (pesada hidrostática).

Los parámetros que se pueden calcular son los siguientes:

I. Porosidad abierta:

$$
\mathrm{n}_{\mathrm{o}}(\%)=\frac{\mathrm{M}_{\mathrm{S}}-\mathrm{M}_{\circ}}{\mathrm{M}_{\mathrm{S}}-\mathrm{M}_{\mathrm{h}}} \times 100
$$

2. Densidad aparente

$$
\mathrm{d}_{\mathrm{ap}}=\frac{\mathrm{M}_{\circ}}{\mathrm{M}_{\mathrm{s}}-\mathrm{M}_{\mathrm{h}}}\left(\mathrm{g} / \mathrm{cm}^{3}\right)
$$

3. Contenido de agua a saturación

$$
\mathrm{W}_{\mathrm{s}}=\frac{\mathrm{M}_{\mathrm{S}}-\mathrm{M}_{\circ}}{\mathrm{M}_{\circ}} \times 100
$$

Donde:

Mo Peso seco

$M_{h}$ Peso saturado sumergido (pesada hidrostática)

$M_{s} \quad$ Peso saturado al aire.

\section{ABSORCIÓN LIBRE DE AGUA. IMBIBICIÓN}

El coeficiente de absorción o grado de impregnación relativa de un material poroso, es el volumen de agua presente en los poros de ese material después de una completa inmersión a presión atmosférica durante un tiempo definido en relación con el volumen total de poros. El objeto de este ensayo es determinar la capacidad natural de la muestra para absorber agua.

La absorción libre de agua depende de las características del material y del tiempo de inmersión; así para tiempos de inmersión más grandes el comportamiento de los materiales en general es más homogéneo. Dado que en muchos tipos de piedra el peso constante no se alcanza jamás, se calcula el coeficiente de saturación, $S_{i}$, como la relación entre el volumen de agua absorbida por la muestra después de la inmersión y el volumen total de los poros abiertos.

El contenido de agua en 24 horas es un parámetro que está incluido en la norma ISSR (1979) con la denominación Índice de vacío. El contenido en agua en 48 horas la norma ASTM (1978) la denomina Absorción de agua. La norma RILEM (1980) la emplea para la determinación del coeficiente de saturación conocido como el coeficiente de Mishwald, en este caso la inmersión es gradual durante el primer día. El contenido en agua para tiempos largos permite alcanzar los valores de equilibrio, la norma ICR (I98I) la denomina capacidad de absorción.

\section{Procedimiento de ensayo}

Las probetas se sumergen en una bandeja con agua destilada sobre un soporte para conseguir el menor contacto de la probeta con la superficie, a temperatura constante $\left(21 \pm 2^{\circ} \mathrm{C}\right)$ durante el tiempo necesario. Se va determinando el peso húmedo de las probetas, $M_{i}$, en función del tiempo, eliminando el agua en exceso con un paño húmedo; la precisión de la pesada debe ser de 0,0lg. Tras cada pesada se sumergen nuevamente en agua. Deberán realizarse pesadas más frecuentes en la primera fase del ensayo.

La duración del ensayo debe ser, al menos, de 7 días. Si se observa que la variación de peso en 24 horas es superior al $1 \%$, se debe prolongar el ensayo.

Resultados. Gráficamente se expresa el contenido en agua (\% peso) en función del tiempo (figura I). 
Fig. 1. Contenido en agua (\% peso) en función del tiempo.

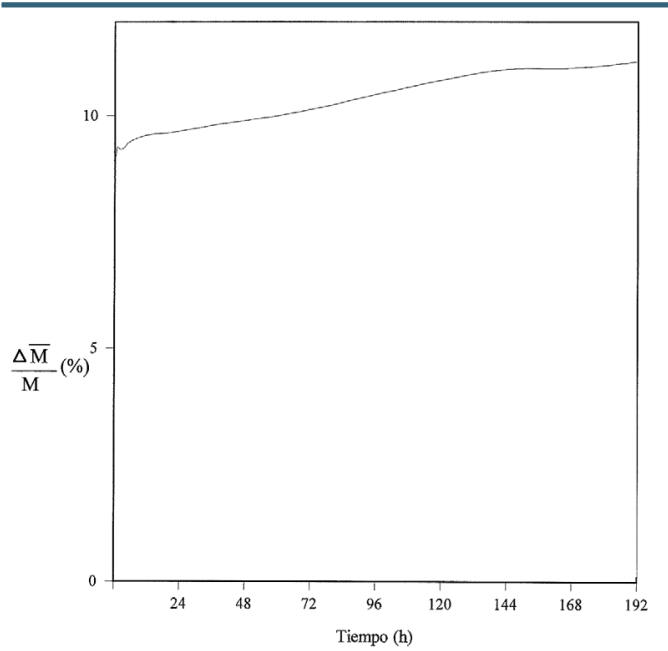

Fig. 2. Incremento de peso por superficie en función de la raíz cuadrada del tiempo

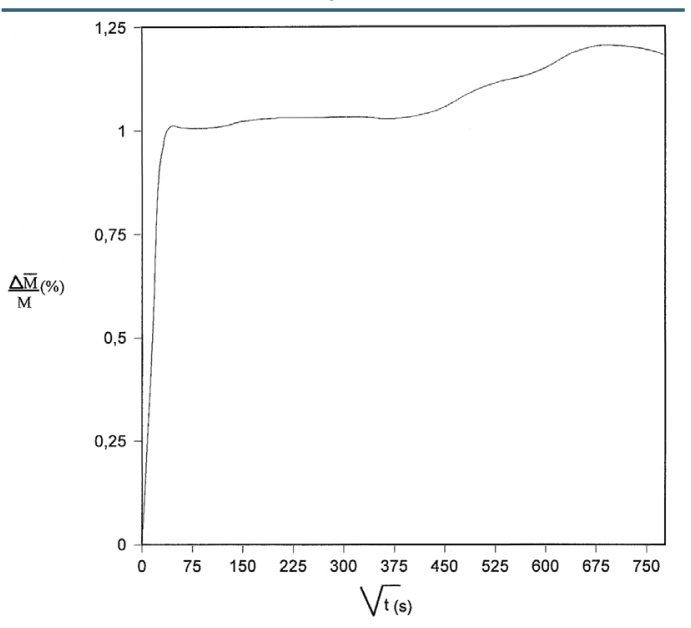

Experimentalmente se observa que la variación de $M_{i}$ respecto al tiempo en los primeros momentos es lineal, calculando la pendiente de esta recta se obtiene el coeficiente de absorción hídrica o velocidad de absorción característico de cada material. A partir de un determinado valor de tiempo en todos los casos se produce una variación de la pendiente llegando a hacerse la curva asintótica para un valor W que prácticamente coincide con la saturación máxima $W(\max$.$) .$

La cantidad de agua absorbida $\Delta M / M(\%)$ por la muestra en el tiempo $T_{i}$ se calcula con la siguiente formula:

$$
\mathrm{W}_{\mathrm{i}}=\frac{\Delta \mathrm{M}}{\mathrm{M}}\left(\frac{\circ}{\circ}\right)=\frac{\mathrm{M}_{\mathrm{i}}-\mathrm{M}_{\mathrm{O}}}{\mathrm{M}_{\mathrm{O}}}-\mathrm{x100}
$$

Los intervalos de pesada suelen seguir una progresión logarítmica, son muy frecuentes al comienzo del ensayo y se van espaciando posteriomente.

Se puede calcular el coeficiente de saturación como

$$
\mathrm{S}_{\mathrm{i}}=\frac{\mathrm{M}_{\mathrm{i}}-\mathrm{M}_{\mathrm{O}}}{\mathrm{M}_{\mathrm{S}}-\mathrm{M}_{\mathrm{O}}}
$$

donde:

$M_{i} \quad$ Peso en el tiempo $t_{i}$

$M_{s}$ Peso saturado obtenido en el ensayo de absorción a vacío

Mo Peso seco

\section{EXPANSIÓN VOLUMÉTRICA POR ABSORCIÓN DE AGUA}

Algunos materiales durante la absorción de agua desarrollan tensiones que son puestas de manifiesto por la expansión volumétrica que experimentan. La repetición periódica de este proceso, puede producir fisuraciones en el material, lo cual es de sumo interés en los procesos de alteración.

Este comportamiento es atribuido a presiones de succión de origen no muy bien conocido, entre las distintas causas apuntadas las de mayor incidencia corresponden a fenómenos de absorción y adsorción de los minerales arcillosos.

El ensayo consiste en determinar el hinchamiento que experimenta una muestra no confinada durante la absorción de agua a partir de la medida de su expansión lineal según tres direcciones ortogonales. En este caso se determina la expansión por absorción de agua en función del tiempo. La duración del ensayo es de 8 a 10 días, la temperatura debe estar en torno a $21^{\circ} \mathrm{C}$.

Se determina la longitud inicial $l_{0}$ y la muestra se coloca en una cubeta con un extensómetro mecánico (sensibilidad I $\mu \mathrm{m}$ ) y se añade agua hasta cubrir sus 3/4 partes. Se va determinando el incremento de longitud en función del tiempo $\Delta \mathrm{l}(\mathrm{t})$ en \%. Las muestras deben ser de forma prismática 70×30×30 (mm).

Los valores de $\xi(\Delta \mid(t))$, dilatación lineal, se representan frente al tiempo t. La curva que se obtiene es la curva de hinchamiento.

$$
\xi=\frac{\Delta l \theta)}{b} \times 100
$$

$\xi_{\max }$ es el valor de equilibrio.

\section{ABSORCIÓN CAPILAR}

La absorción capilar es el método fundamental de transporte capilar de sales hacia el interior de la roca debido al efecto de la ósmosis y foresis. El movimiento de los líquidos a través de un sólido poroso se realiza en sentido de menor densidad o menor concentración de sales. Los deterioros sufridos por los materiales relacionados con el ascenso capilar están relacionados con los procesos de cristalización de sales y de agua el interior de los poros capilares, así como por la acción de hidrólisis, hidrataciones, oxidaciones, etc. de los componentes minerales de la piedra. Esta absorción no solamente se produce por el agua del subsuelo sino en todo contacto del agua con la superficie pétrea, ya sea por el agua de condensación, de lluvia, etc. El agua líquida en contacto con el sistema poroso de las 
rocas ve favorecida su succión por el efecto de capilaridad directamente relacionado con el tamaño de poro. La fuerza de succión depende de la naturaleza de la superficie del diámetro de poro. La altura de ascenso capilar puede ser calculada de forma teórica por la ecuación (Ley de Javin):

$$
\begin{aligned}
& \mathrm{H}=\frac{2 \cos \theta}{r \boldsymbol{t}} \\
& \mathrm{H} \text { : altura de ascensión rupilar } \\
& \theta \text { : ángulo de contacto líquido-sólido } \\
& r \text { : rádio de poro } \\
& \gamma \text { viscosidad }
\end{aligned}
$$

En este ensayo se puede medir la variación de la altura alcanzada por el agua absorbida en función del tiempo, así como se puede determinar el incremento de la masa, refiriéndola a la superficie de contacto con el líquido, también en función del tiempo.

Descripción del Ensayo. Las probetas deben tener sección constante a lo largo de su altura y planeidad de sus bases. Para determinar la altura alcanzada por el agua es aconsejable utilizar probetas cilíndricas o prismáticas, con la dimensión mayor perpendicular a la superficie de absorción. Las condiciones ambientales del ensayo en torno a $20^{\circ} \mathrm{C}$ de temperatura, humedad relativa de $50 \%$ y una duración de al menos 7 días.

Las probetas secas se sitúan en una bandeja en cuya base se coloca una delgada capa de material poroso (arena) sobre la que se coloca otra de papel de flitro (al menos de I cm de grosor) y se añade agua destilada hasta el nivel del papel de filtro, manteniendo constante el mismo a lo largo de todo el ensayo.

Tras intervalos regulares de tiempo ( I hora, 8 horas, 24 horas, 48 horas) se pesan eliminando con la ayuda de un paño húmedo el agua en exceso de la superficie inferior. Igualmente se determina la altura alcanzada por el agua, calculando un valor medio con los datos de las cuatro caras

Representación de los resultados. La curva de absorción capilar (figura 2) representa el incremento de peso por superficie en función de la raíz cuadrada del tiempo. Se representa gráficamente $\Delta M / S$, siendo

$$
\begin{aligned}
& M_{i} \text { peso en el tiempo tis } \\
& M_{0} \text { peso seco } \\
& S \text { superficie de absorción }
\end{aligned}
$$

Curva de penetración capilar: Se representa la altura ascendida, $\mathrm{H}_{\mathrm{i}}$, en función del la raiz cuadrada del tiempo.

\section{ABSORCIÓN DE AGUA A BAJA PRESIÓN}

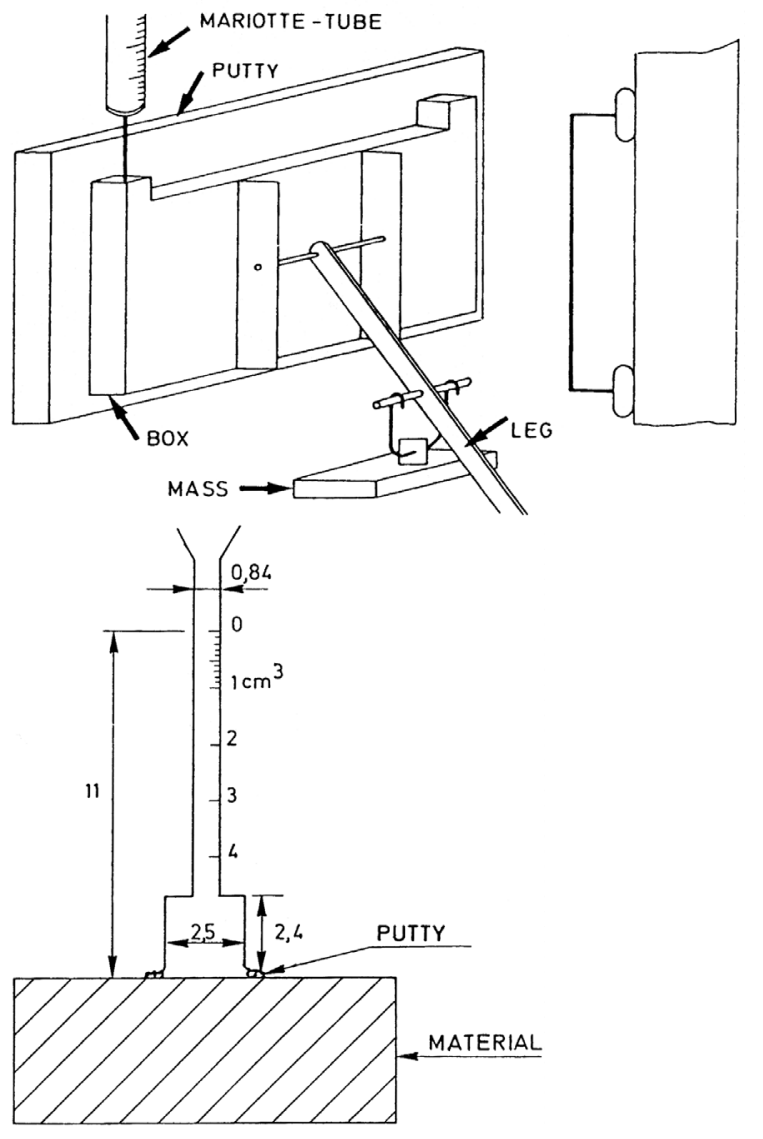

Fig. 3.

Fig. 4.

Este ensayo mide el volumen de agua que absorbe a baja presión un material poroso a través de la superficie en función del tiempo, refiriéndolo a la unidad de superficie. Estos ensayos se realizan en in situ directamente sobre la superficie (muros, sillares etc.) del edificio.

Existen básicamente dos métodos: método de la caja y método de la pipa. Los dispositivos empleados en los dos método aparecen en las figuras 3 y 4.

La cantidad de agua que penetra en la pared se va controlando directamente con un tubo graduado en $\mathrm{cm}^{3}$. Durante los primeros minutos la medida se realiza cada 15 segundos, después cada minuto hasta 5 minutos. El ensayo se realiza durante 30 minutos.

Se calcula la cantidad de agua absorbida por unidad de superficie y se representa frente al tiempo.

\section{Bibliografía}

FORT GONZÁLEZ, R. (1996). "El agua en los materiales pétreos: comportamiento hídrico" Degradación y conservación del Patrimonio

PRADA LÓPEZ, J.L. “Caracterización de formas y procesos de alteración, observadas en piedra de construcción de edad Miocénica del área Monumental romana de Tarragona". Tesis Doctoral Dpto. De Geoquímica, petrología y Prospección geológica. Universidad de Barcelona.
DE LA TORRE LÓPEZ, M.J. (1996). "Propiedades hídricas" Técnicas de diagnóstico aplicadas a la conservación de los materiales de construcción en edificios históricos. Cuadernos técnicos. Edita IAPH, Junta de Andalucía. pp.66-7I. 